\title{
THE EXPONENT OF CERTAIN FINITE $p$-GROUPS
}

\author{
by I. O. YORK \\ (Received 15th January 1990)
}

In this paper, for $\mathbf{R}$ a commutative ring, with identity, of characteristic $p$, we look at the group $\mathbf{G}(\mathbf{R})$ of formal power series with coefficients in $\mathbf{R}$, of the form

$$
\sum_{i=0}^{\infty} a_{i} x^{i}, a_{0}=0, a_{1}=1
$$

and the group operation being substitution. The results obtained give the exponent of the quotient groups $\mathbf{G}_{n}(\mathbf{R})$ of this group, $n \in \mathbb{N}$.

1980 Mathematics subject classification (1985 Revision): $20 \mathrm{D} 15$.

\section{Introduction}

In this paper we will deal with the group $\mathbf{G}(\mathbf{R})$ of formal power series

$$
f(x)=x+a_{2} x^{2}+a_{3} x^{3}+\ldots
$$

where the coefficients are elements of a commutative ring $\mathbf{R}$, with identity, and the group operation is substitution. A study of this group is carried out in [3] and also of the groups $\mathbf{G}_{n}(\mathbf{R})$ whose elements can be considered as elements of $\mathbf{G}(\mathbf{R})$ truncated to $n$ terms. Such objects were studied from other points of view in [1]. The groups when $\mathbf{R}$ is a commutative ring, with identity, of characteristic $p$ are studied by the author as due to their large class they can often achieve, or at least approach, bounds on such properties as derived length of classes of $p$-groups studied by other authors. Often the power structure of the groups $G_{n}(R), R$ a commutative ring, with identity, of characteristic $p$,needs to be known in order to show that they satisfy the conditions on the p-groups to which the bounds refer. Hence the purpose of this paper is to find the exponent of the groups $\mathbf{G}_{n}(\mathbf{R})$ for all $n \in \mathbb{N}$ and for $R$ a commutative ring, with identity, of characteristic $p$.

1. The exponent of the groups $G_{n}(R)$, where $R$ is a commutative ring, with identity, of characteristic $p, p \geqq 3$

We start with some definitions and notation. If $\alpha \in \mathbf{G}(\mathbf{R}), \alpha \neq x$ and $\alpha=\sum_{i=1}^{\infty} a_{i} x^{i}$, $a_{1}=1, a_{i}=0$ (for $2 \leqq i<n$ ) and $a_{n} \neq 0$ we say $\operatorname{deg}(\alpha)=n$. Also define the subset $\mathbf{K}_{r}$ of $\mathbf{G}(\mathbf{R})$ 
by $\mathbf{K}_{r}=\{\alpha \in \mathbf{G}(\mathbf{R})$ : $\operatorname{deg}(\alpha)>r\}$, then $\mathbf{K}_{r}$ is a normal subgroup of $\mathbf{G}(\mathbf{R})$, the proof of which is in [1], and we define $G_{n}(R)$ as the quotient group $G(R) / K_{n}$.

The following notation will be used in this paper: If $\alpha \in \mathbf{G}(\mathbf{R})$ then $\alpha^{(m)}$ is the $m$ th iterate of $\alpha$, while $\alpha^{m}$ is the $m$ th power of $\alpha$. Furthermore we shall denote by $\mathbf{R}[[x]]$ the algebra over $\mathbf{R}$ of all formal power series with indeterminant $x$ and coefficients in $\mathbf{R}, \mathbf{a}$ commutative ring with identity.

Lemma 1. ([2, Theorem 2.5]) If $\mathbf{R}$ has characteristic $p$, then $\mathbf{K}_{r}^{(p)} \subseteq \mathbf{K}_{r p}$.

The question now asked is: what more can we say when $\mathbf{R}$ is a commutative ring, with identity?

Observation 2. Let $\mathbf{R}$ be a commutative ring, with identity and $\alpha \in \mathbf{G}(\mathbf{R})$. Then the map $\eta: \mathbf{R}[[x]] \rightarrow \mathbf{R}[[x]]$ given by $g(x) \mapsto g(\alpha)$, is an $\mathbf{R}$-algebra automorphism. Further $\eta$ preserves the ideal $(x)$.

The proof of Observation 2 is standard and hence is omitted.

Notation. Let $z_{n}$ be defined as $z_{n}=p^{n}+p^{n-1}+\cdots+p+2$.

Lemma 3. Let $\alpha \in \mathbf{G}(\mathbf{R})$ and $\eta$ be as defined in Observation 2. Then on the basis $x, x^{2}, \ldots, x^{m}, \ldots$ of $(x)$, the action of $\eta$ is given by:

$$
\left(\begin{array}{c}
x \\
x^{2} \\
x^{3} \\
\vdots
\end{array}\right) \mapsto \mathbf{M}\left(\begin{array}{c}
x \\
x^{2} \\
x^{3} \\
\vdots
\end{array}\right)
$$

where $\mathbf{M}=\left(m_{i, j}\right)$ is the matrix such that $m_{i, j}=$ coefficient of $x^{j}$ in $\alpha^{i}$.

Proof. As by Observation $2 \eta$ preserves $(x)$, we know the action of $\eta$ on the given basis elements of $(x)$ is in the form of the lemma for some $\mathbf{M}$. By the definition of $\eta, \eta\left(x^{j}\right)=\alpha^{j}$. Thus we need to prove that the $j$ th row of the vector

$$
\mathbf{M}\left(\begin{array}{c}
x \\
x^{2} \\
x^{3} \\
\vdots
\end{array}\right) \text { is } \alpha^{j}
$$

Now by the definition of $M$ the $j$ th row in this vector is 


$$
\sum_{i=1}^{\infty}\left(\text { coefficient of } x^{i} \text { in } \alpha^{j}\right) x^{i}=\alpha^{j}
$$

Lemma 4. If $\alpha_{1}, \alpha_{2} \in \mathbf{G}(\mathbf{R})$, and the maps $\eta_{i}: \mathbf{R}[[x]] \rightarrow \mathbf{R}[[x]]$ are given by $g(x) \mapsto$ $g\left(\alpha_{i}\right)(i=1,2)$, and if $\mathbf{M}_{i}$ is the matrix of Lemma 3 corresponding to $\alpha_{i},(i=1,2)$ then $\mathbf{M}_{1} \mathbf{M}_{2}$ is the matrix corresponding to $\alpha_{1}\left(\alpha_{2}\right) \in \mathbf{G}_{n}(\mathbf{R})$.

Proof. Now we have that if $\eta: \mathbf{R}[[x]] \rightarrow \mathbf{R}[[x]]$ is given by $g(x) \mapsto g\left(\alpha_{1}\left(\alpha_{2}\right)\right)$ then

$$
\begin{aligned}
\eta\left(x^{i}\right) & =\left(\alpha_{1}\left(\alpha_{2}\right)\right)^{i} \\
& =\alpha_{1}^{i}\left(\alpha_{2}\right) \\
& \left.=\sum_{j=1}^{\infty} \sum_{k=1}^{\infty}\left(\text { coefficient of } x^{k} \text { in } \alpha_{1}^{i}\right) \text { (coefficient of } x^{j} \text { in } \alpha_{2}^{k}\right) x^{j} \\
& =i \text { th row in the vector } \mathbf{M}_{1} \mathbf{M}_{2}\left(\begin{array}{c}
x \\
x^{2} \\
x^{3} \\
\vdots
\end{array}\right)
\end{aligned}
$$

because using the definition of $\mathbf{M}_{1}$ and $\mathbf{M}_{2}$

$$
\left.\left(\mathbf{M}_{1} \mathbf{M}_{2}\right)_{i, j}=\sum_{k=1}^{\infty}\left(\text { eoefficient of } x^{k} \text { in } \alpha_{1}^{i}\right) \text { (coefficient of } x^{j} \text { in } \alpha_{2}^{k}\right)
$$

Lemma 5. Let $\mathbf{M}$ be defined as in Lemma 3, define $\Delta$ by $\mathbf{M}=I+\Delta$ where $I$ is the identity matrix, then

$$
\left(\Delta^{p^{m}}\right)_{1, d}=\sum_{j} \Delta_{1, j_{1}} \Delta_{j_{1}, j_{2}} \ldots \Delta_{j_{1}, d}
$$

where $l=p^{m}-1$ and $\mathbf{j}=\left\{\left(j_{1}, \ldots, j_{l}\right): 2 \leqq j_{1}<j_{2}<\cdots<j_{l} \leqq d-1\right\}$.

Further if $d \neq \equiv(\bmod p)$ and the set $\left(j_{1}, \ldots, j_{l}\right)$ gives a non-zero term in the right hand side of (1) then $j_{i} \neq 0(\bmod p)(1 \leqq i \leqq l)$.

Proof. Equation (1) follows directly from the definitions. We prove by contradiction that if $d \neq 00(\bmod p)$ then for a term in the right hand side of (1) to be non-zero it is necessary that,

$$
j_{i} \not \equiv 0(\bmod p)\left(1 \leqq i \leqq p^{m}-1\right)
$$

Thus we assume that in a non-zero term in the right hand side of $(1), j_{i} \equiv 0(\bmod p)$ 
for some $i, 1 \leqq i \leqq p^{m}-1$, and show by an inductive argument that this implies that $d \equiv 0$ $(\bmod p)$ which is the required contradiction.

Examination of (1) gives that to complete the inductive argument and obtain the required contradiction we only need prove that

$$
r \equiv 0(\bmod p), s \neq 0(\bmod p) \Rightarrow \Delta_{r, s} \equiv 0(\bmod p) .
$$

Now we know,

$$
\alpha^{p t}=\sum_{j=1}^{\infty} m_{p t, j} x^{j}
$$

It is clear that $\theta: \alpha \mapsto \alpha^{p}$ is a endomorphism of the ring of formal power series. So we obtain,

$$
\begin{aligned}
\alpha^{p t}=\left(\alpha^{t}\right)^{p} & =\sum_{j=1}^{\infty}\left(m_{t, j} x^{j}\right)^{p} \\
& =\sum_{j=1}^{\infty} m_{t, j}^{p} x^{p j}
\end{aligned}
$$

Thus we conclude that,

$$
m_{p t, j}= \begin{cases}m_{i, k}^{p} & \text { if } j=p k \\ 0 & \text { if } j \neq \equiv 0(\bmod p)\end{cases}
$$

Therefore as,

$$
\Delta_{r, s}=\text { coefficient of } x^{s} \text { in } \alpha^{r}
$$

we have the contradiction.

Theorem 6. Let $\mathbf{R}$ be a commutative ring, with identity of characteristic $p \geqq 3$ and $z_{m}$ as defined above. Then for $n<z_{m}$ the exponent of $\mathbf{G}_{n}(\mathbf{R})$ is at most $p^{m}$.

Proof. (In fact the proof of the following equivalent statement: If $\mathbf{R}$ is a commutative ring, with identity, of characteristic $p \geqq 3$ and $z_{m}$ is as defined above. Then for all $\alpha \in \mathbf{G}(\mathbf{R}), \alpha^{\left(p^{m)}\right)} \in \mathbf{K}_{z_{m}-1}$.)

Let $\alpha \in \mathbf{G}(\mathbf{R})$

The map $\eta: \mathbf{R}[[x]] \rightarrow \mathbf{R}[[x]]$, given by

$$
g(x) \mapsto g(\alpha)
$$


is an R-algebra automorphism, by Observation 2 .

By Lemma 3 the action of $\eta$ on the basis $x, x^{2}, \ldots, x^{n}, \ldots$ of $(x)$ is given by:

$$
\left(\begin{array}{c}
x \\
x^{2} \\
x^{3} \\
\vdots
\end{array}\right) \mapsto M\left(\begin{array}{c}
x \\
x^{2} \\
x^{3} \\
\vdots
\end{array}\right)
$$

where $\mathbf{M}=\left(m_{i j}\right)$ is the matrix such that $m_{i j}=$ coefficient of $x^{j}$ in $\alpha^{i}$.

By Lemma 4 the action of the R-algebra automorphism of $\mathbf{R}[[x]]$, given by $g(x) \mapsto g\left(\alpha^{(r)}\right)$ on the basis $x, \ldots, x^{n}, \ldots$ is given by:

$$
\left(\begin{array}{c}
x \\
x^{2} \\
x^{3} \\
\vdots
\end{array}\right) \mapsto \mathbf{M}^{r}\left(\begin{array}{c}
x \\
x^{2} \\
x^{3} \\
\vdots
\end{array}\right)
$$

Put $\mathbf{M}=I+\Delta$, where $I$ is the identity matrix, so that $\mathbf{M}^{p^{k}} \equiv I+\Delta^{p^{k}}(\bmod p)$. Now (3) gives us that:

$$
\left(M^{p^{k}}\right)_{1, i}=\text { coefficient of } x^{i} \text { in } \alpha^{\left(p^{k}\right)}
$$

Hence in order to prove the theorem we require that,

$$
\left(\Delta^{p^{m}}\right)_{1, i} \equiv 0(\bmod p) \text { for all } 1 \leqq i \leqq z_{m}-1 \text {. }
$$

We now proceed to prove this by induction on $m$. For $m=0:(\Delta)_{1, i} \equiv 0(\bmod p)$ for all $1 \leqq i \leqq z_{0}-1=1$ as $\Delta$ has 0 on and below the main diagonal. Now we assume for $j<m$ that

$$
\left(\Delta^{p^{j}}\right)_{1, i} \equiv 0(\bmod p) \text { for all } 1 \leqq i \leqq z_{j}-1
$$

Thus using the inductive hypothesis and (4) we have that

$$
\alpha^{\left(p^{m-1}\right)} \in \mathbf{K}_{z_{m-1}-1}
$$

and thus by Lemma 1 that

$$
\alpha^{\left(p^{m}\right)} \in \mathbf{K}_{p\left(z_{m-1}-1\right)}
$$

Hence by again using (4), in order to complete the inductive step it is only now necessary to prove that: 


$$
\left(\Delta^{p^{m}}\right)_{1, z_{m}-1} \equiv 0(\bmod p)
$$

By Lemma 5,

$$
\left(\Delta^{p^{m}}\right)_{1, z_{m}-1}=\sum_{j} \Delta_{1, j_{1}} \Delta_{j_{1}, j_{2}} \ldots \Delta_{j_{1}, z_{m}-1}
$$

where $l=p^{m}-1$ and

$$
\mathbf{j}=\left\{\left(j_{1}, j_{2}, \ldots, j_{p^{m-1}}\right): 2 \leqq j_{1}<j_{2}<\cdots<j_{p^{m-1}} \leqq z_{m}-2\right\} .
$$

Now the number of integers in the range 2 to $z_{m}-2$ divisible by $p$ is $p^{m-1}+p^{m-2}+$ $\cdots+1$, hence the number of integers in this range not divisible by $p$ is $p^{m}-2$. By definition, $z_{m}-1 \equiv 1 \not \equiv 0(\bmod p)$ so we know by Lemma 5 that for a non-zero term in the right hand side of (5) we are required to choose an ordered set of integers $\left(j_{1}, \ldots, j_{p^{m-1}}\right)$ such that

$$
2 \leqq j_{1}<j_{2}<\cdots<j_{p^{m}-1} \leqq z_{m}-2 \text { and } j_{s} \neq 0(\bmod p)\left(1 \leqq s \leqq p^{m}-1\right)
$$

which is not possible as there are only $p^{m}-2$ integers in the range 2 to $z_{m}-2$ not divisible by $p$. Hence $\left(\Delta^{p^{m}}\right)_{1, z_{m}-1} \equiv 0(\bmod p)$, which completes the inductive step.

We thus have the required result that,

$$
\left(\Delta^{p^{m}}\right)_{1, i} \equiv 0(\bmod p) \text { for all } 1 \leqq i \leqq z_{m}-1 \text {. }
$$

Having obtained a bound for the exponent we now consider the powers of a specific element in order to show that the bound is achieved.

Theorem 7. Let $z_{k}$ be as defined above, $\mathbf{R}$ be any commutative ring, with identity, of characteristic $p, p \geqq 3$. Then for all $k \in \mathbb{N}$, the $p^{k}$ th iterate of $x+x^{2}$ over $\mathbf{R}$ is $x+x^{z_{k}}+\cdots$.

Proof. We consider the map $\eta$ defined in Observation 2 in the special case of $\alpha=x+x^{2}$. Then as before putting $\mathbf{M}=I+\Delta$, where $I$ is the identity matrix, so that $\mathbf{M}^{p^{k}} \equiv I+\Delta^{p^{k}}(\bmod p)$, where $\mathbf{M}$ is the matrix defined in Lemma 3 in the special case $\alpha=x+x^{2}$.

By Theorem 6 we have that $\left(x+x^{2}\right)^{\left(p^{k}\right)} \in \mathbf{K}_{z_{k}-1}$ and thus by the definition of $\mathbf{M}$ and $\Delta$,

$$
\left(\Delta^{p^{k}}\right)_{1, q} \equiv 0(\bmod p)\left(2 \leqq q \leqq z_{k}-1\right)
$$

It is clear by definition that

$$
\Delta_{i, j}= \begin{cases}\left(\begin{array}{c}
i \\
j-1
\end{array}\right) & \text { if } 1<j \leqq 2 i \\
0 & \text { otherwise. }\end{cases}
$$


In this case it is thus obvious that all non-zero terms in the right hand side of (1) with $d=z_{k}$ have $j_{1}=2$. So

$$
\left(\Delta^{p^{k}}\right)=\sum_{j^{\prime}} \Delta_{2, j_{2}} \ldots \Delta_{j l, z_{k}}
$$

where $j^{\prime}=\left\{\left(j_{2}, \ldots, j_{l}\right): 3 \leqq j_{2}<j_{3}<\cdots<j_{l} \leqq z_{k}-1\right\}$ and $l=p^{k}-1$.

As $z_{k} \equiv 2 \neq 0(\bmod p)$, by Lemma 5 we obtain that for a non-zero term in the right hand side of (6) we must have,

$$
j_{s} \not \equiv(\bmod p) \text { for all } 2 \leqq s \leqq p^{k}-1 .
$$

As we are required to choose an ordered set $\left(j_{2}, \ldots, j_{p^{k}-1}\right)$ of integers such that $3 \leqq j_{2}<j_{3}<\cdots<j_{p^{k}-1} \leqq z_{k}-1$, and there are $p^{k}-2$ integers in the range 3 to $z_{k}-1$ not divisible by $p$, there can only be one non-zero term; which is

Case (a): $p>3$

$\left(\Delta^{p^{k}}\right)_{1, z_{k}}=$

$$
\left(\begin{array}{l}
2 \\
1
\end{array}\right)\left(\begin{array}{l}
3 \\
1
\end{array}\right) \cdots\left(\begin{array}{c}
p-2 \\
1
\end{array}\right)\left(\begin{array}{c}
p-1 \\
2
\end{array}\right)\left(\begin{array}{c}
p+1 \\
1
\end{array}\right) \cdots\left(\begin{array}{c}
2 p-2 \\
1
\end{array}\right)\left(\begin{array}{c}
2 p-1 \\
2
\end{array}\right)\left(\begin{array}{c}
2 p+1 \\
1
\end{array}\right) \cdots\left(\begin{array}{c}
d p-1 \\
2
\end{array}\right)\left(\begin{array}{c}
d p+1 \\
1
\end{array}\right)
$$

where

$$
z_{k}=d p+2, d \in \mathbb{N} .
$$

Now we know that for $f \in \mathbb{N}$ that,

$$
\begin{aligned}
\left(\begin{array}{c}
f p+2 \\
1
\end{array}\right)\left(\begin{array}{c}
f p+3 \\
1
\end{array}\right) \ldots\left(\begin{array}{c}
f p+p-2 \\
1
\end{array}\right) & =(f p+2) \ldots(f p+p-2) \\
& \equiv 2.3 \ldots(p-2)(\bmod p) \\
& \equiv 1(\bmod p)(\text { By Wilson's Theorem) } \\
\text { Further }\left(\begin{array}{c}
f p-1 \\
2
\end{array}\right)\left(\begin{array}{c}
f p+1 \\
1
\end{array}\right) & \equiv 1(\bmod p) \text { for all } f \in \mathbb{N} .
\end{aligned}
$$

Case (b): $p=3$

$$
\left(\Delta^{p^{k}}\right)_{1 . z_{k}}=\left(\begin{array}{l}
4 \\
1
\end{array}\right)\left(\begin{array}{l}
5 \\
2
\end{array}\right)\left(\begin{array}{l}
7 \\
1
\end{array}\right)\left(\begin{array}{l}
8 \\
2
\end{array}\right) \cdots\left(\begin{array}{c}
3 d-1 \\
2
\end{array}\right)\left(\begin{array}{c}
3 d+1 \\
1
\end{array}\right)
$$

where

$$
z_{k}=3 d+2, d \in \mathbb{N} .
$$

Now we know that for $f \in \mathbb{N}$ that, 


$$
\left(\begin{array}{c}
3 f+1 \\
1
\end{array}\right) \equiv 1(\bmod p)
$$

and

$$
\left(\begin{array}{c}
3 f+2 \\
2
\end{array}\right) \equiv 1(\bmod p)
$$

Hence the result follows in both cases.

Combining Theorems 6 and 7 we readily obtain the following theorem.

Theorem 8. Let $\mathbf{R}$ be a commutative ring, with identity, of characteristic $p \geqq 3$ and $z_{m}$ be as defined as above. Then for $z_{m-1} \leqq n<z_{m}$ the exponent of $\mathbf{G}_{n}(\mathbf{R})$ is $p^{m}$.

\section{The exponent of the groups $G_{n}(R)$, where $R$ is an integral domain of characteristic 2}

This case differs substantially from the case of odd $p$. For example the exponent is the order of $x+x^{3}$ rather than $x+x^{2}$ for $\mathbf{R}=\mathbb{Z}_{2}$ and is the order of $x+x^{2}+a x^{3}$ (where $a \in \mathbf{R}, a \neq 0, a \neq 1$ ) when $\mathbf{R} \neq \mathbb{Z}_{2}$. As this case is of less interest from the point of view of the applications indicated in the introduction we merely summarize.

Theorem 9. The exponent of $\mathbf{G}_{n}\left(\mathbb{Z}_{2}\right)$ is $2^{m}$, where $2^{m}+1 \leqq n<2^{m+1}+1$ for $n \geqq 5$.

Theorem 10. The exponent of $\mathbf{G}_{n}(\mathbf{R})$, where $\mathbf{R}$ is an integral domain of characteristictwo and $\mathbf{R} \neq \mathbb{Z}_{2}$ is $2^{m}$, where $2^{m} \leqq n<2^{m+1}$, i.e. $m=\left[\log _{2} n\right]$.

Acknowledgements. I wish to express my gratitude to R. W. K. Odoni who by proving Theorem 7 in the special case of $k=1$ provided the underlying method used in this paper. I would also like to thank the referee for his comments which gave a simpler and shorter proof of Lemma 5 , as well as pointing out that the working in Section 1 applied not only to fields of characteristic $p$ but to all the rings studied in the section.

\section{REFERENCES}

1. I. N. Baker, Permutable power series and regular iteration, J. Austral. Math. Soc. 2 (1961-62), 265-294.

2. S. A. Jennings, Substitution groups of formal power series, Canad. J. Math. 6 (1954), 325-340.

3. D. L. Johnson, The group of formal power series under substitution, J. Austral. Math. Soc. 45 (1988), 296-302.

UNIVERSITY OF NOTTINGHAM

University Park

NOTTINGHAM

NG7 2RP 